\title{
A INTERAÇÃO DE USUÁRIOS EM ESPAÇOS LIVRES: SIMULAÇÕES COM AGENTES AUTÔNOMOS
}

\section{USER INTERACTION IN OUTDOOR SPACE: SIMULATIONS USING AUTONOMOUS AGENTS}

\author{
Carlos Eduardo Verzola Vaz ${ }^{1}$ \\ Universidade Federal de Santa Catarina, Florianópolis, SC, Brasil, carlos.vaz@ufsc.br \\ João Pedro Schneider ${ }^{2}$ \\ Universidade Federal de Santa Catarina, Florianópolis, SC, Brasil, joaopedrosndr@gmail.com \\ Marcus Vinícius da Silva ${ }^{3}$ \\ Universidade Federal de Santa Catarina, Florianópolis, SC, Brasil, marcusvinicdasilva@gmail.com \\ Renata Nunes Brochi Rodrigues 4 \\ Universidade Federal de Santa Catarina, Florianópolis, SC, Brasil, renata.brochi@gmail.com \\ Arthur Stofella ${ }^{5}$ \\ Universidade Federal de Santa Catarina, Florianópolis, SC, Brasil, stofella42@gmail.com
}

\begin{abstract}
Resumo
O objetivo deste trabalho é apresentar os resultados de uma pesquisa que busca desenvolver simulações para auxiliar arquitetos e urbanistas a planejar intervenções em espaços livres. Este documento contém os métodos utilizados para coletar dados de dinâmica do usuário em espaços abertos e fechados, os padrões observados durante o processo de coleta de dados e um protótipo de uma simulação implementada usando uma biblioteca de agentes autônomos no ambiente de desenvolvimento Processing. Durante a pesquisa são estudadas as dinâmicas humanas em átrios de edifícios de sala de aula e nos espaços livres próximos ao Restaurante Universitário da Universidade Federal de Santa Catarina. As simulações, inicialmente desenvolvidas em duas dimensões, possibilitam modificar a dinâmica dos agentes pela inserção de novos objetos em um cenário inicial e pela alteração de parâmetros ambientais, tais como, temperatura umidade, ou situações de céu coberto ou encoberto. A simulação apresentada neste trabalho foca na formação de diferentes grupos de pessoas, o fluxo espacial do usuário e o comportamento em relação a outros objetos presentes em um espaço livre, tais como árvores e bancos. Os resultados desta pesquisa nos ajudarão a desenvolver ferramentas para prever como os padrões de comportamento humano em espaços livres e ajudar estudantes a desenvolver uma compreensão de como pequenas intervenções podem mudar a dinâmica de um lugar.
\end{abstract}

Palavras-chave: Simulação. Dinâmica de usuários. Agentes de jogos. Espaços livres.

\begin{abstract}
The aim of this paper is to present the results of research that seeks to develop simulations to assist architects and urban designers in planning interventions in outdoor spaces. This document will present the methods used to collect data of user dynamics and occupation in open and closed spaces, the patterns observed during the data collection process and a prototype of a simulation implemented using an autonomous agent library from Processing IDE. Open spaces around the University restaurant and atriums of classroom blocks of the Federal University of Santa Catarina are used as case studies. The simulations, represented in two dimensions, were developed to modify the agent's dynamic by the insertion of new objects in an initial scenario and alteration of environmental parameters, such as temperature or situations of cloud or uncloud sky. The simulation presented in this paper focuses on different conformation of groups of people, the spatial user flow and the behavior concerning other objects present in an outdoor space, such as trees and benches. The results from this research will assist us to develop tools to predict how patterns of human behavior in outdoor spaces and to assist students in developing their understanding of how small interventions can change the dynamic of a place.
\end{abstract}

Keywords: Simulation. User dynamic. Game agents. Outdoor spaces.

How to cite this article:

VAZ, Carlos Eduardo Verzola et al.. A interação de usuários em espaços livres: simulações com agentes autônomos. PARC Pesquisa em Arquitetura e Construção, Campinas, SP, v. 10, p. e019001, jan. 2019. ISSN 1980-6809. Disponível em: <https://periodicos.sbu.unicamp.br/ojs/index.php/parc/article/view/8651586>. Acesso em: 27 dez. 2018. doi:https://doi.org/10.20396/parc.v10i0.8651586. 


\section{Introdução}

De acordo com Gehl (2006), as atividades que ocorrem nos espaços livres podem ser divididas em duas categorias principais:

1. Atividades necessárias: são as atividades consideradas compulsórias, tais como ir à escola, ao trabalho ou esperar o ônibus. Essas atividades ocorrem ao longo do ano, independentemente das condições ou do ambiente externo;

2. Atividades opcionais: essa categoria inclui atividades como pegar um ar fresco, aproveitar a vida, sentar em um local e tomar um banho de sol. Essas atividades ocorrem nos espaços livres apenas quando as condições climáticas são consideradas ótimas, quando o clima e o lugar são convidativos.

O autor explica que a relação entre as atividades necessárias e opcionais e o planejamento do espaço físico é especialmente importante, pois a maioria das atividades recreativas dependem das condições físicas dos ambientes externos. Quando os espaços livres apresentam baixa qualidade, apenas atividades estritamente necessárias ocorrem. Por outro lado, quando estes apresentam alta qualidade, além das atividades necessárias pode-se verificar a ocorrência de atividades opcionais.

Durante o processo de projeto, arquitetos e urbanistas buscam constantemente prever como os usuários vão se comportar e qual será o caráter do lugar que está sendo projetado. Normalmente, o objetivo é qualificar bem os espaços para atrair as pessoas, tornando-os constantemente vivos, levando a um padrão de atividades necessárias e opcionais. Mesmo guiado pela experiência, algumas vezes os projetistas falham nessa busca, induzido a ocorrência de situações inesperadas, às vezes aceitáveis, enquanto outras indesejáveis. Atualmente, já é possível simular como um ambiente construído irá se comportar em relação a parâmetros ambientais ou energéticos (LAMBERTS; HENSENAND, 2011). Contudo, há uma carência em relação a simulações capazes de reproduzir o comportamento humano. O modelo de um sistema baseado em eventos criado por Simeone e Kalay (2012) foi uma das primeiras tentativas de se implementar um sistema capaz de simular atividades humanas, indo além das simulações de multidões ou fluxo de usuários (BRAUN et. al, 2003).

Em ambientes internos, eventos usualmente resultam de uma atividade humana. Contudo, em espaços livres eles são influenciados por outros eventos, tais como o canto de pássaros, o movimento da água, dos carros etc. O arranjo espacial entre objetos e o padrão de eventos são responsáveis por definir o caráter do lugar, que, por sua vez, pode atrair ou repelir usuários. O problema é que milhares de eventos ocorrem simultaneamente em espaços livres, tornando impraticável representar todos os eventos do mundo real e tentar prever o caráter do lugar. Isto torna o processo de implementação de simulações computacionais um problema. Atualmente, inúmeras plataformas utilizadas por arquitetos e urbanistas para "renderizar" e gerar vídeos de projetos utiliza tecnologias de jogos e de agentes autônomos. Contudo, na maioria destes sistemas as regras de comportamento implementadas são elementares, gerando assim cenários que não refletem a realidade. Como resultado, as imagens geradas são visualmente realistas, mas o sistema de regras implementado no algoritmo pode ser considerado frágil.

O objetivo deste trabalho é introduzir os resultados de uma pesquisa que visa desenvolver um método para descrever o caráter de espaços livres e identificar os parâmetros que podem afetar e atrair pessoas a desenvolver uma atividade. Além disso, a pesquisa objetiva implementar simulações com agentes autônomos de modo a validar os dados coletados em um cenário real. A hipótese da pesquisa é que uma adequada 
descrição do lugar pode levar a simulações capazes de prever a dinâmica de um espaço livre e auxiliar arquitetos e urbanistas a compreender o impacto de suas decisões no processo de projeto. Neste trabalho é desenvolvido um método para coleta de dados, representação de padrões de uso de espaços e uma simulação computacional utilizando agentes autônomos com o objetivo de reproduzir as atividades realizadas por usuários em espaços livres.

\section{Fundamentação}

A fundamentação abordará de forma breve quatro temas importantes para o desenvolvimento do trabalho. O primeiro relaciona-se com a percepção dos usuários em relação ao espaço e como eles coletam dados e interpretam informações do espaço durante um processo de tomada de decisão. O segundo aspecto importante a ser tratado refere-se às relações entre os usuários e como se formam os diferentes grupos de indivíduos. O terceiro tema abordado relaciona-se à definição de um ferramental (gramática da forma e lógica de primeira ordem) para a descrição das diferentes situações observadas no espaço, possibilitando-se assim a posterior implementação dos padrões observados em uma simulação dinâmica. Finalmente, o último aspecto abordado trata dos conceitos básicos de simulações com agentes autônomos.

\section{Percepção do ambiente - a relação entre indivíduo e espaço}

De acordo com Kaplan e Kaplan (1989), para qualquer ambiente há combinações e arranjos de elementos que o usuário precisa constantemente decodificar antes de tomar uma decisão. Os autores explicam que constantemente estamos capturando uma quantidade significativa de dados do ambiente. Segundo Friedenberg e Silverman (2006), após observarmos uma cena, mesmo que por um período de tempo muito breve, uma representação é criada em nossa memória sensorial. Essa representação inclui informações que computamos da cena e, a partir delas, temos a capacidade de gerar um significado para um dado sinal sensorial ou evento percebido. Tal significado é, posteriormente, avaliado a partir de experiências anteriores, armazenadas em nossa memória de longo prazo, responsável por formar compreensão de como o mundo funciona. Esse processo, segundo Kaplan e Kaplan (1989), permite que os seres humanos avaliem situações presentes e futuras permitindo que sejam compreendidas como boas ou ruins, agradáveis ou desagradáveis. Como resultado, os autores explicam que os seres humanos estão capacitados a prever eventos por meio de sua imaginação, criando planos alternativos ou produzindo instruções ou conselhos para outra pessoa. Esse processo não se baseia apenas em informações que podem ser extraídas de forma imediata, mas também em dados que estão latentes no ambiente e que os seres humanos julgam com tal facilidade que muitas vezes não estão cientes do fato de que isso esteja ocorrendo.

A coleta contínua de dados e seu armazenamento na memória de longo prazo colaboram no condicionamento de nosso olhar em relação a determinadas características do ambiente, o que ajuda no processo de identificação de possíveis funcionalidades no espaço, onde o prazer e a beleza também podem constituir um tipo de utilidade que pode nos oferecer, de certo modo, felicidade (GRAHNA; STIGSDOTTER, 2010). A capacidade de identificar as funcionalidades que o ambiente pode proporcionar é definida por Gibson (1986) pelo termo affordance. Segundo o autor, affordance pode ser traduzido como sendo o que a estrutura ambiental oferece a um organismo para que este realize uma atividade, baseando-se nas relações entre o meio ambiente e o organismo. Ou seja, quais são as características de um objeto ou ambiente que possibilitam a um indivíduo identificar intuitivamente a sua funcionalidade. Quanto 
menos definida a funcionalidade, maior a chance de o organismo identificar ou utilizar o espaço de diferentes formas. Por essa razão, muitas vezes, alguns arranjos espaciais proporcionam diferentes funcionalidades, pois os usuários tendem a se basear em experiências anteriores e a explorar diferentes possibilidades de uso. Por exemplo, a visão de água ou o arranjo espacial de uma árvore e um banco em um gramado fornece informações que podem ser interpretadas e, a partir de experiências passadas, fornecem a informação necessária para uma tomada de decisão. Segundo Goffman (1971), esse processo muitas vezes envolve algum tipo de maximização de ganhos, ainda que sob condições de incerteza ou riscos. Isso fornece um sentido no qual o ator (agente) pode ser considerado como sendo "racional" e também uma perspectiva eticamente neutra do processo de julgamento relativo à conveniência de diferentes cursos de ação.

Contudo, apesar das experiências passadas, serem responsáveis por moldar a percepção de cada indivíduo em relação ao espaço, há uma tendência para um padrão de comportamento entre seres humanos, mesmo existindo, como afirmam Veitch e Arkkelin (1995), uma diferença em relação a como as pessoas percebem o ambiente, segundo suas experiências, diferenças culturais e traços de personalidades.

Interação entre usuários - a relação entre indivíduos no espaço

Além da relação do indivíduo com o ambiente, os usuários relacionam-se entre si, formando grupos de diferentes tamanhos. No processo de composição de grupos de usuários alguns aspectos relacionados à disposição espacial das pessoas e à forma com que estas se comportam entre si devem ser considerados.

Em relação à disposição espacial dos usuários, Kendon (1990) explica que o indivíduo tem um espaço social no qual tipicamente direciona sua atenção e manipula objetos. $\mathrm{O}$ espaço social de um indivíduo é definido em relação à parte inferior do corpo. Uma formação em $\mathrm{F}$ (ou face a face) se constitui quando duas ou mais pessoas se organizam de forma que seus espaços sociais se sobreponham. Essa justaposição gera um espaço convexo vazio cercado pelas pessoas envolvidas na interação social onde cada participante olha para dentro e nenhuma pessoa externa é permitida nessa região. Ao redor dos participantes de uma interação, na parte externa, forma-se um espaço denominado $\mathrm{R}$, em que não são realizadas atividades, mas no qual os indivíduos mantêm a atenção.

Figura 1 - Diferentes formações F: a) formação em L; b) Formação face a face; c) Formação lado a lado; d) formação semicircular; e) Formação retangular

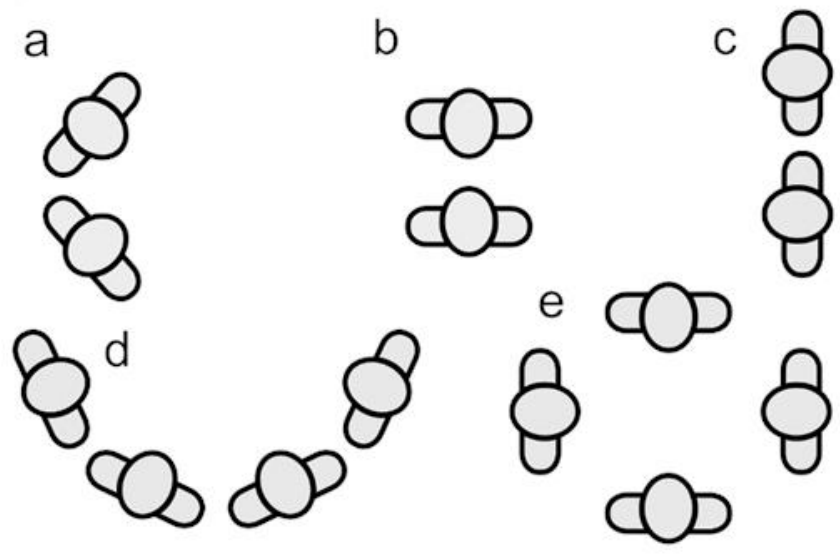

Fonte: Marshall, Rogers e Pantidi (2011). 
Lawson (2001) define, em sua pesquisa na área da psicologia ambiental, os padrões de comportamento durante a socialização entre indivíduo s (confrontamento, colaboração e coexistência). $O$ autor define o confrontamento como a situação em que duas pessoas estão em conflito e simbolicamente veem o mundo de ângulos opostos. O papel de colaboração acontece quando duas pessoas estão conversando e compartilham da mesma visão de mundo, tendendo a se organizar de modo a dividir uma mesma perspectiva. Finalmente, o papel de coexistência ocorre quando dois indivíduos compartilham o mesmo espaço, no entanto, não estão desenvolvendo nenhuma atividade em conjunto.

\section{Representação de padrões em regras}

A gramática da forma é um método de análise, reprodução ou geração de novas linguagens compositivas criado por Stiny e Gips (1972), desenvolvido a partir das teorias da gramática generativa de Noan Chomsky e dos sistemas de produção de Post. Essa metodologia de construção de composições visuais representa uma sistemática de produção formal, baseada em um processo algorítmico, capaz de gerar composições baseadas em um vocabulário de formas primitivas e suas relações espaciais. A concatenação das regras de uma gramática em diferentes combinações permite a construção de composições pertencentes a uma mesma linguagem ou estilo. Como o propósito desta pesquisa não é o estudo aprofundado da gramática da forma, esses componentes serão descritos de modo informal, apenas para que o leitor entenda o seu funcionamento. Na lista abaixo são enumerados alguns dos elementos básicos de uma gramática da forma:

- Formas: Elementos que podem ser tanto bidimensionais como tridimensionais. Podem ser elaboradas em um espaço finito de tempo, com um número finito de elementos que podem ser linhas retas, curvas conectadas ou desconectadas e por um conjunto de linhas que podem gerar formas fechadas ou faces.

- Vocabulário de formas: É um conjunto de formas que fazem parte de uma gramática.

- Forma inicial: A partir desta forma será iniciado o processo de aplicação das regras contidas na gramática.

- Regras: Uma regra é composta por duas formas separadas por uma seta. Para que possa ser aplicada, a forma presente do seu lado esquerdo deve ser identificada em uma composição que está sendo derivada. Em seguida, essa forma será substituída pela que está à direita da seta na regra.

A gramática da forma pode ser utilizada para descrever desde linguagens simples, com poucas regras, como a apresentada abaixo, até sistemas mais complexos, formado por inúmeras regras. A disposição de usuários no espaço ao longo de um período de tempo também pode ser descrita por regras de uma gramática da forma. A disposição das pessoas em um espaço muda ao longo do tempo. Em um curto período de tempo não é possível verificar um padrão de distribuição, contudo, quanto maior o período de observação, é possível notar um padrão de uso, o que implica a existência de regras subjacentes que são dependentes das intenções dos usuários, da forma como os usuários se relacionam, das características espaciais e dos parâmetros ambientais. De certa maneira, pode-se também definir esta distribuição como uma linguagem, passível de ser compreendida.

A verificação desses tipos de padrões, por meio da utilização de um processo de observação sistemático, foi realizada por Whyte (1971) em seu projeto de pesquisa sobre a vida nos espaços livres de Nova York. Em conjunto com um grupo de pesquisadores, 
o autor realizou, por meio da utilização de entrevistas e gravações em timelapse, um levantamento com o objetivo de compreender a dinâmica de uso desses espaços. Nesse trabalho foram verificados os espaços de sentar e a influência do sol, do vento, das árvores e da água no processo de escolha do usuário. No caso de Whyte (1971), esses dados foram transpostos em diferentes tipos de gráficos e em mapas e a explicação dos fatores que alteram a dinâmica de uso do espaço foi expressada por meio de texto.

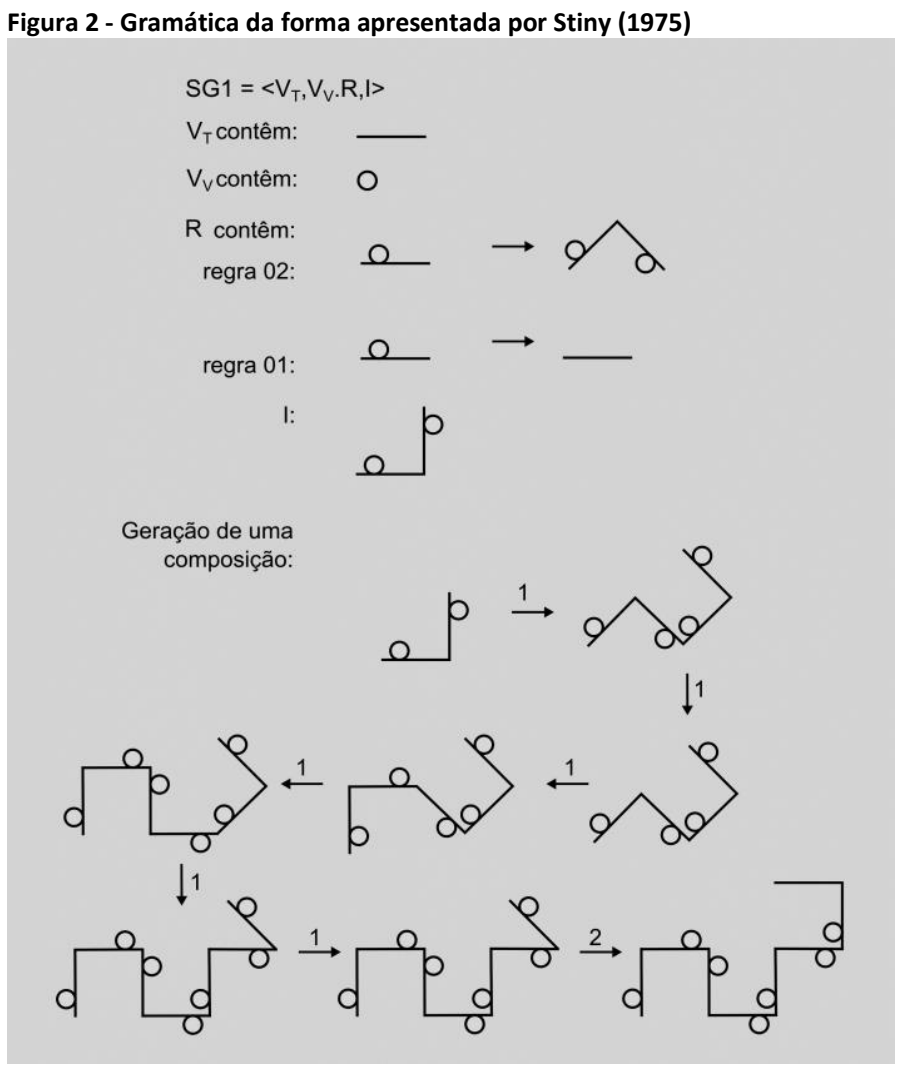

Fonte: Stiny (1975).

Nesta pesquisa, uma gramática da forma esquemática será utilizada para expressar os padrões observados pelos pesquisadores. Segundo Stiny (2006), o processo de desenvolvimento de uma gramática de formas envolve a identificação e classificação de propriedades essenciais e acidentais de uma família de projetos. Somente propriedades essenciais devem estar presentes na gramática, no entanto, nem sempre é necessário representar todas elas. $\mathrm{O}$ autor afirma que o número de propriedades essenciais pode variar de acordo com o objetivo da gramática. Uma gramática da forma pode ser construída para descrever exatamente todos os recursos de uma linguagem. No entanto, também é possível construí-la de forma concisa, criando regras que representam apenas suas principais características.

Além da gramática da forma, sentenças de lógica de primeira ordem foram utilizadas para representar os aspectos mais dinâmicos e não visuais, como métricas ambientais (temperatura, luminosidade etc.) ou fenômenos que dão qualidade ao local (sons, movimento etc.). Mitchell (1990) emprega esse tipo de formalismo para exemplificar como os críticos de arquitetura vão além e combinam suas observações com fatos e regras sobre o mundo da construção para derivar condições que não são evidentes. $A$ base de conhecimento da crítica não é apenas moldada por aspectos de forma arquitetônica ou características estilísticas. Michell (1990) explica que também pode incluir fatos e regras derivadas da economia e áreas de ciências sociais (um crítico pode se preocupar com a relação entre construção e comportamento humano). 


\section{Agentes autônomos}

Buckland (2004) explica que há uma diferença para a área acadêmica e de jogos. No primeiro caso, não há limitação em relação ao tempo de processamento e recursos, sendo que a implementação visa simular a inteligência humana (strong $\mathrm{Al}$ ) ou resolver problemas do mundo real (weak $\mathrm{Al}$ ). No caso da aplicação da inteligência artificial na área de jogos, o objetivo é criar uma ilusão de inteligência. Apesar deste ser um trabalho científico, esta abordagem é mais compatível, pois assim como na área de jogos, é necessário trabalhar com uma quantidade limitada de ciclos de processamento e de memória, sendo que, além disso, cada usuário utiliza plataformas com diferentes especificações.

Segundo Shiffman (2012), no final dos anos de 1980 o cientista da computação Craig Reynolds desenvolveu comportamentos de direção algorítmica para agentes animados. De acordo com o autor, esses comportamentos permitiram que entidades individuais navegassem em seus ambientes digitais de maneira "realista", com estratégias para fugir, vagar, chegar, perseguir, evadir etc. Shiffman (2012) explica que implementar um único agente a partir desses comportamentos pode ser considerada uma tarefa simples e a construção de um sistema de múltiplos personagens que se orientam de acordo com regras simples possibilita a emergência de simulações com diferentes níveis de complexidade.

\section{Metodologia}

A pesquisa foi realizada em três etapas diferentes. Na primeira fase foi definida uma metodologia para a coleta de dados utilizada como ponto de partida para compreender o funcionamento de duas áreas selecionadas como estudo de caso (um átrio de um conjunto de salas de aula e um espaço livre). O objetivo era compreender como se dava a dinâmica de funcionamento desses espaços, concentrando a atenção no padrão de atividades dos usuários e sua interação com diferentes objetos presentes no espaço. Como resultado, foi utilizada a observação naturalística como método para obter informações sobre o comportamento das pessoas em ambos os estudos de caso, variando apenas a forma como os dados eram coletados. As observações se concentraram na compreensão das dinâmicas de uso em duas escalas diferentes. A primeira delas, em relação ao usuário, tiveram como foco os seguintes aspectos:

i. Como as pessoas se dispõem em relação umas às outras no espaço;

ii. Qual o papel do usuário na atividade.

A segunda escala observada durante a etapa de coleta de dados relaciona-se à dinâmica de movimento em ambos os estudos de caso. Em outras palavras, como se dá o fluxo de pessoas no espaço, qual a duração das atividades, em que lugares as pessoas se encontram e quais são as características desses espaços. A metodologia utilizada por Gehl e Svarre (2013) para estudar a dinâmica de espaços livres na cidade foi utilizada como referência. $\mathrm{O}$ autor explica que contar é uma ferramenta amplamente utilizada em estudos da vida pública. Em princípio, tudo pode ser contado, fornecendo números, o que permite fazer comparações do antes e depois, entre diferentes áreas geográficas ou ao longo do tempo. O movimento de pessoas cruzando ou permanecendo em um espaço podem ser representados como linhas de movimento ou manchas em um plano da área em estudo. Durante essa fase, os pesquisadores observaram principalmente a densidade de movimento, o tempo de permanência e os objetos utilizados pelos usuários. 
Durante o processo de observação três instrumentos diferentes foram utilizados para coletar dados: imagens, esboços e anotações. A partir dessas informações mapas temáticos (fluxos, comportamentos etc.) foram montados tentando representar as dinâmicas de uso nos locais estudados. Além das observações naturalísticas, dados de funcionamento dos espaços também foram obtidos a partir de outras pesquisas que utilizam o campus como estudo de caso.

$\mathrm{Na}$ segunda fase da pesquisa a informação coletada durante a observação foi organizada e analisada pelos pesquisadores com o objetivo de identificar diferentes padrões de atividade e fluxo, relacionando-as com:

i. A organização do usuário no espaço: com base nas pesquisas de Lawson (2001) sobre psicologia espacial, os padrões de atividade foram organizados segundo o número de participantes e sua postura corporal para identificar situações de confronto, colaboração ou coexistência;

ii. Fluxos de usuário: foram identificadas as principais rotas, horários e intensidades de movimento, tanto de pedestres e como de ciclistas;

iii. Arranjos espaciais: nesse caso foram identificados as características espaciais e os objetos que constantemente estão presentes nos locais em que uma atividade era realizada. Por exemplo, em uma determinada área, estudantes podem constantemente se acomodar para ler sob a copa de uma árvore. A árvore, nesse caso, é o objeto que está presente no espaço e apresenta atributos (geométricos, qualitativos, etc.) que a tornam um elemento importante no lugar.

Além da coleta de dados sobre a configuração espacial e os eventos humanos que sistematicamente ocorrem nos locais, os pesquisadores também observaram como eventos não humanos e métricas ambientais impactaram a dinâmica de uso dos lugares estudados, atraindo ou não usuários.

O material coletado tanto para a análise do ambiente interno quanto externo foram organizados em imagens, vídeos, textos descritivos e mapas capazes de retratar os dois estudos de caso nas diferentes escalas.

Nessa mesma etapa o material coletado foi traduzido em regras capazes de descrever de forma simplificada a dinâmica de uso dos espaços estudados. Essa representação foi feita utilizando regras esquemáticas de gramática da forma para explicitar padrões de ocupação e, no caso de parâmetros não visuais, tais como temperatura e situações de céu coberto ou encoberto, sentenças de lógica de predicado.

Na terceira fase da pesquisa as regras obtidas após a modelagem foram implementadas em uma simulação a partir de uma plataforma livre de programação utilizando como referência conceitos de agentes autônomos.

Durante essa etapa foram definidos como e quais objetos e parâmetros deveriam ser reproduzidos com o objetivo de simular os processos observados nas situações reais. Ao longo da pesquisa os diferentes cenários de simulação foram comparados com a realidade com o objetivo de aprimorar as regras de comportamento do sistema. Os cenários criados para a simulação podem ser modificados pela adição de novos objetos (árvores e bancos) e alterações de parâmetros ambientais (temperatura, luminosidade etc.) possibilitando, assim, modificações na dinâmica do "lugar". Como será visto, a implementação foi realizada considerando o caso dos espaços livres. 


\section{Observação nos espaços fechados}

Nesta etapa da pesquisa foram realizadas observações para dois tipos de espaços: espaços livres e fechados. No primeiro caso, o objetivo era excluir a ação direta dos parâmetros ambientais sobre a dinâmica de uso e ocupação. Os locais para a realização deste estudo foram cinco átrios de diferentes blocos de sala de aulas pertencentes a vários departamentos da Universidade Federal de Santa Catarina. Todos os espaços foram construídos a partir de um mesmo projeto, sendo que há poucas variações entre eles (Figura 3).

Durante o período aproximado de dois meses foi observado e registrado o processo de ocupação desses espaços. A partir dessa análise foram verificadas situações relacionadas principalmente com a densidade de uso e da organização corporal entre os usuários. No primeiro caso, verificou-se que a maior parte dos usuários não utilizava o átrio interno em sua parte central, permanecendo principalmente nas bordas, próximo às áreas de corredor que dão acesso às salas de aula. A maior parte das pessoas não permanece no espaço, utilizando-o como área de passagem. Essa situação é observada também nas pesquisas de Whyte (1971), que concluiu que em espaços amplos os usuários tendem a ocupar as suas bordas.

Quando o espaço estudado era ocupado, os usuários permaneciam por curtos períodos de tempo, sendo que apenas uma parte sentava-se. Como o edifício estudado era um bloco de salas de aula, havia um ciclo relacionado com o início das aulas. Em relação ao posicionamento dos usuários, foi possível verificar padrões de organização nos locais de sentar. Ao estudar esses espaços, constatou-se que as pessoas que se sentavam sozinhas escolhiam o local mais distante da outra pessoa já posicionada, geralmente na extremidade oposta do banco que ali estava e quase sempre utilizavam o celular nessa situação. Quando as pessoas se reuniam em grupos, normalmente permaneciam localizadas perto das saídas ou locais de maiores fluxos. Nos pontos de encontro em que havia bancos, quando os grupos eram superiores a duas pessoas, uma ou mais permanecia de pé, respeitando assim o espaço social dos demais membros. A Figura 3 apresenta os padrões observados traduzidos para regras simplificadas.

Figura 3 - (1) Algumas das imagens coletadas durante o processo de coleta de dados no espaço livre; (2) mapeamento dos fluxos, das situações de formação de grupos e as atividades realizadas; (3) Padrões observados

1 | Imagens levantamento

2| Mapeamento
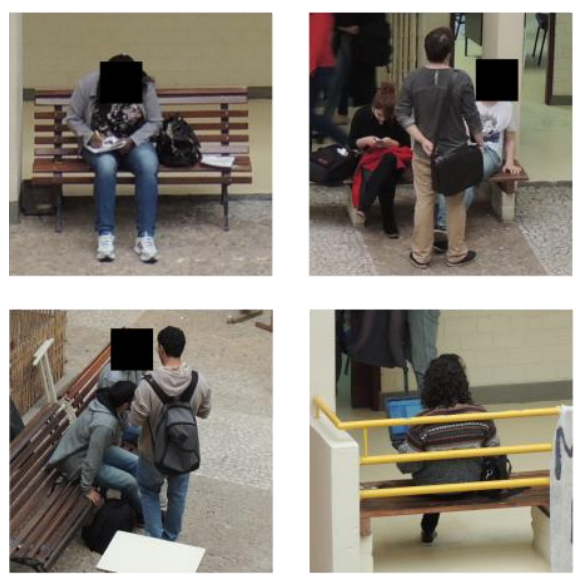

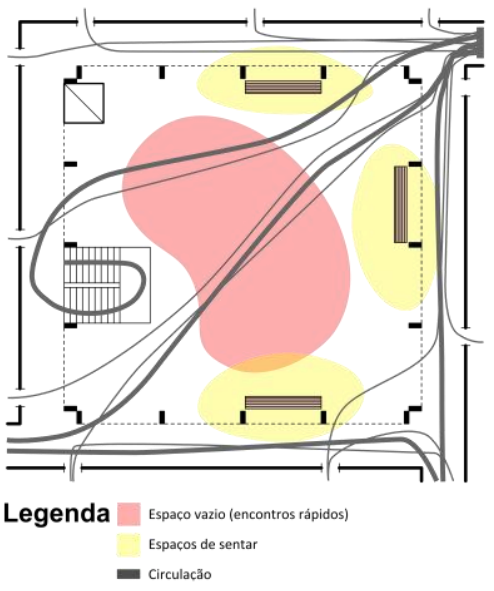

3 | Padrões de comportamento



Fonte: Os autores.

\section{Observação nos espaços abertos}

A partir do levantamento em espaços fechados realizou-se também o estudo de um espaço livre, aplicando-se metodologia semelhante. Como estudo de caso foram 
selecionados os espaços livres próximos ao Restaurante Universitário do mesmo campus.

Esses espaços se tornaram local de encontro para os acadêmicos de diferentes departamentos. Durante o período de aula, forma-se uma fila que pode alcançar mais de 200 metros de comprimento. As áreas gramadas próximas tornaram-se, durante a hora do almoço, espaços de lazer passivo da comunidade do campus (Figura 4).

O processo de observação ocorreu pelo período de três meses, entre maio e julho de 2016. Como esse espaço era formado por uma área bem maior, durante o primeiro mês foi realizado um teste para definir as ferramentas mais adequadas para se coletar os dados. A dificuldade era registrar as diferentes atividades que ocorriam simultaneamente. Sendo assim, optou-se por realizar um registro de vídeo em movimento por todo o comprimento da fila, com o intuito de estudar as dinâmicas que nela ocorriam. Além disso, imagens foram coletadas de qualquer grupo de pessoas próximo à fila que estavam utilizando o gramado ao redor do restaurante.

Como nesse caso fica mais clara a influência das métricas climáticas sobre o uso do espaço, também foi gerado um arquivo com os dados do dia para verificar alterações de padrão de uso por parte dos usuários. Todas as coletas foram realizadas em dias letivos e no mesmo horário (das $12 \mathrm{~h} 15$ às 13h15). A partir desse processo formou-se uma biblioteca com dados sobre a dinâmica do lugar, que possibilitou identificar os padrões de comportamento dos usuários não apenas em relação à configuração espacial e objetos presentes no espaço, mas também em relação às métricas ambientais. Os dados coletados inicialmente foram transpostos em mapas que mostram a dinâmica e o percurso da fila, os lugares escolhidos pelos usuários para se encontrarem e as atividades que eles normalmente faziam (Figura 4 e 5). A partir dos mapas gerados foram identificados alguns padrões de comportamento que foram correlacionados a parâmetros climáticos e elementos presentes no espaço (árvores, bancos, gramado etc.).

Figura 4 - (1) Algumas das imagens coletadas durante o processo de coleta de dados no espaço livre; (2) mapeamento dos fluxos, das situações de formação de grupos e as atividades realizadas; (3) Padrões observados
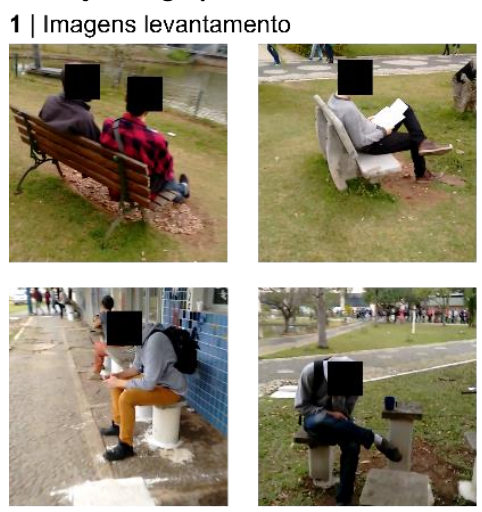

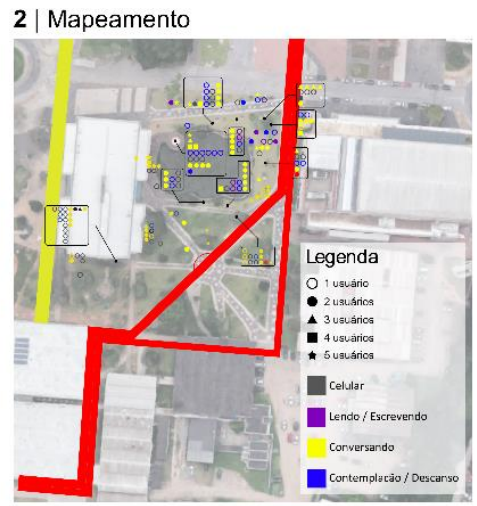

3 | Padrões de comportamento

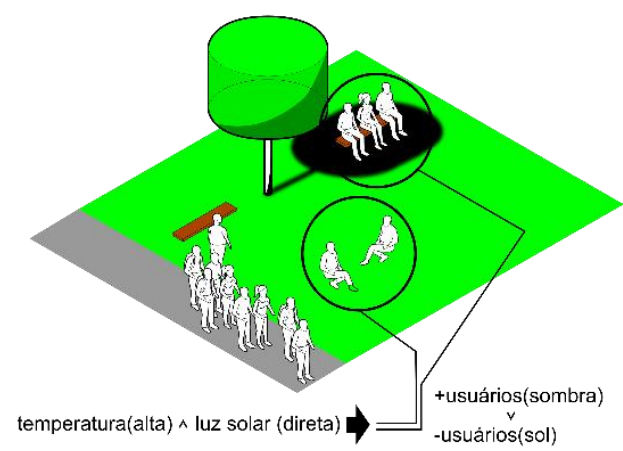

Fonte: Os autores. 
VAZ, Carlos Eduardo Verzola et al..

A interação de usuários em espaços livres: simulações com agentes autônomos

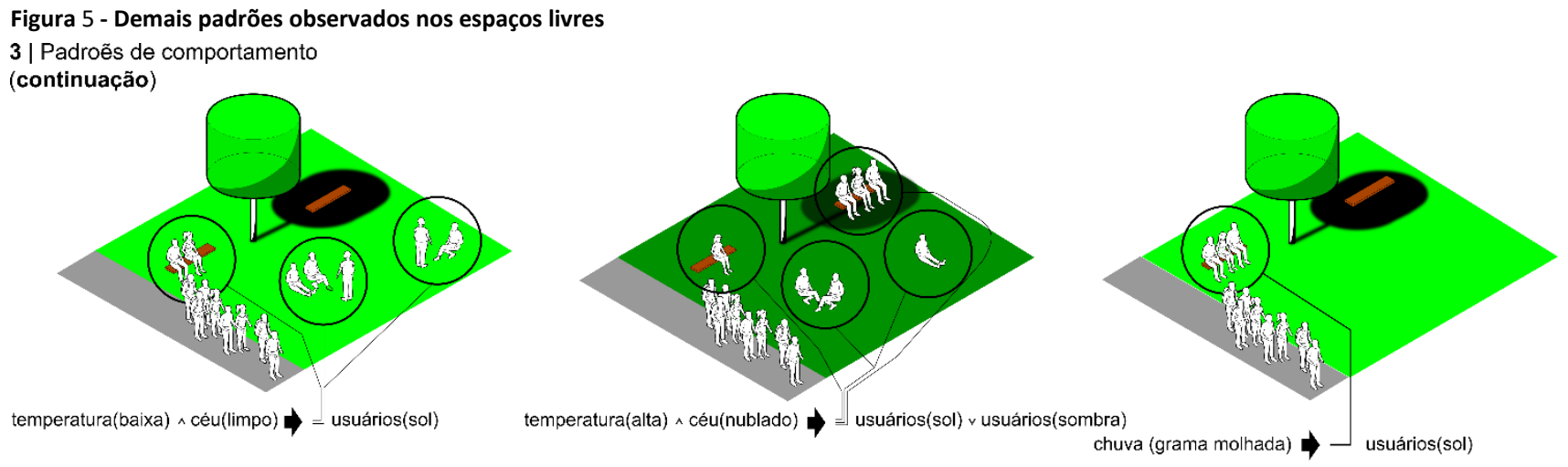

Fonte: Os autores.

Durante o processo de levantamento os pesquisadores também focaram o processo de observação na relação entre os usuários e o objeto árvore. Nesse caso foi possível identificar padrões de disposição, principalmente quando havia o uso de um objeto, tais como celulares ou notebooks pelo indivíduo. Por exemplo, em situações de céu claro, contudo com temperatura baixa, há uma tendência de as pessoas disporem o corpo no sol e o objeto na sombra, na borda sombreada gerada pela copa. Essas regras são apresentadas na Figura 6.

Figura 6 - Padrões de distribuição dos usuários em relação às árvores

1

temperatura(quente)^céu(limpo) $\rightarrow$

temperatura(fria)^céu(limpo) $\rightarrow$

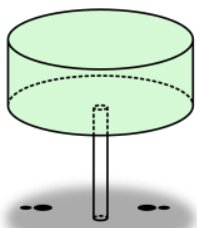

corpo(usuário, sombra)^objeto(usuário, sombra)

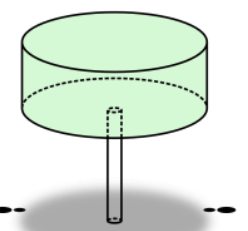

corpo(usuário, sol)^ objeto(usuário, sombra)

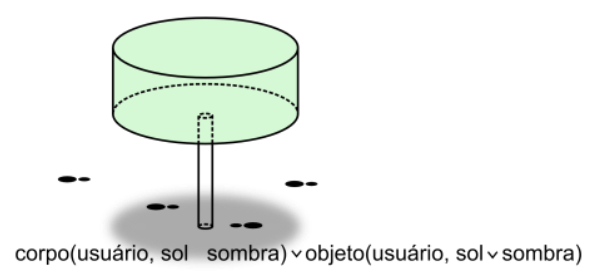

2

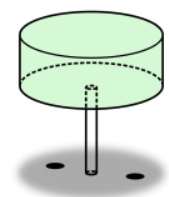

árvore(copa, pequena) $\rightarrow$ usuários $<=2$

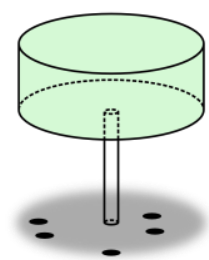

árvore(copa, grande) $\rightarrow$ usuários >=2

Fonte: Os autores.

\section{Simulações}

A partir das observações realizadas tanto nos espaços abertos como nos espaços fechados foi realizada uma simulação computacional visando automatizar os padrões ou regras de comportamentos extraídos dos locais estudados. A implementação foi feita utilizando como referência as atividades identificadas no espaço livre e as alterações observadas a partir da inserção de novos objetos no espaço.

A simulação se concentrou em representar a dinâmica entre as pessoas da fila e os espaços de encontro próximos a ela e a interação com alguns objetos presentes no 
espaço, tais como bancos e árvores. Esses objetos foram escolhidos para serem adicionados ao ambiente virtual, pois eram os que os usuários mais se relacionavam no espaço. Além da relação entre usuários no espaço também foi considerada a alteração climática como fator de alteração da dinâmica do lugar e a variação da posição do sol ao longo do dia, responsável por gerar a posição das sombras no ambiente.

A implementação foi realizada utilizando o ambiente de desenvolvimento livre Processing e uma biblioteca que implementa diferentes funções e entidades relacionadas à inteligência artificial extraídas do livro "Programming Game Al by Example" de Buckland (2004). As ferramentas presentes na biblioteca possibilitaram a implementação de um ambiente em 2D com agentes que se comportam buscando o encontro com outros agentes e por objetos presentes no espaço. $O$ fator de atração (seekOn) é definido por um dos métodos previamente estabelecidos para os agentes (vehicle) da biblioteca para atrair os agentes em relação aos objetos e definir o nível de incidência de formação dos grupos. As situações de encontro entre os usuários, implementadas na simulação (Figura 7), consideram a formação de grupos de duas a quatro pessoas, sendo que a formação desses grupos ocorre de forma dinâmica.

Figura 7 - Situações de formação de grupos, possibilidades de adição ou

subtração de agentes nos grupos e o padrão de distribuição de usuários na fila

\section{Possibilidade de grupos}

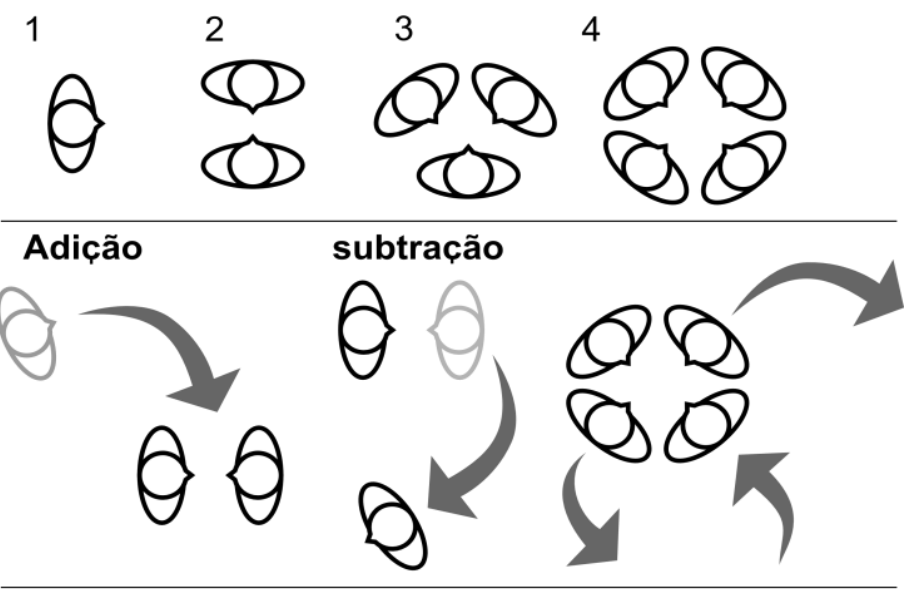

Fila
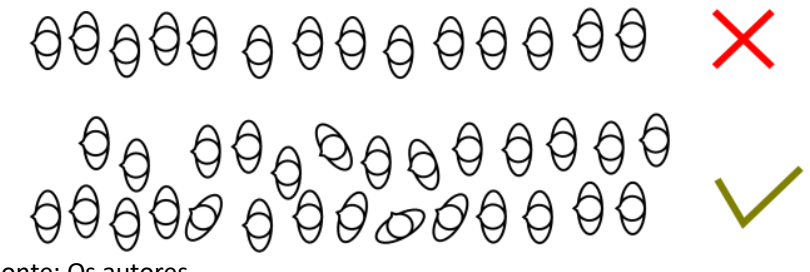

Fonte: Os autores.

O objetivo, neste caso, é simular encontros casuais entre usuários, como foi verificado nos casos de estudo. A Figura 8 mostra o processo de formação dos grupos em uma simulação com um cenário simplificado. As imagens de 1 a 4 ilustram a aproximação dos agentes ao longo do tempo, os agrupamentos e sua posterior separação. Além dos encontros, a fila que se forma ao longo das áreas verdes também faz parte da simulação. O alinhamento dos agentes em fila também se dá pela utilização do método seekOn. Os agentes se enfileiram atraídos por um mesmo ponto, simulando a intenção dos usuários de se direcionarem ao Restaurante Universitário. 
Figura 8 - Implementação da formação de grupos (2 a 3 agentes) no ambiente Processing e indicação complementar para auxiliar na leitura das imagens

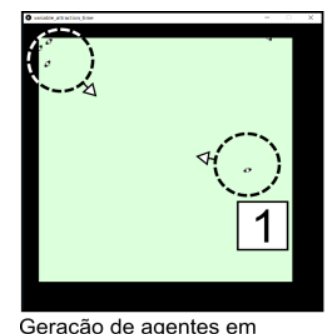

Geração de agentes em

orrible estration nime

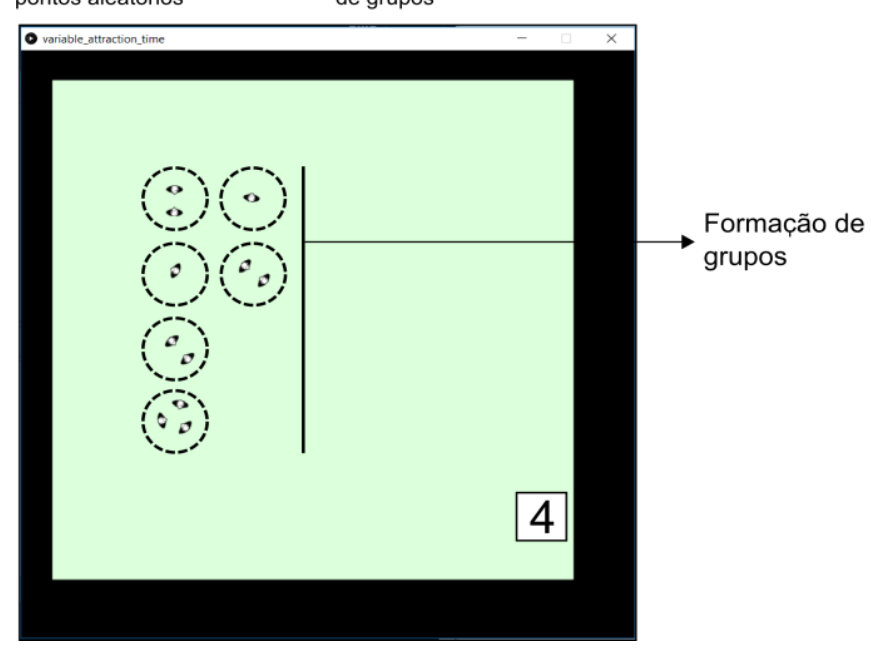

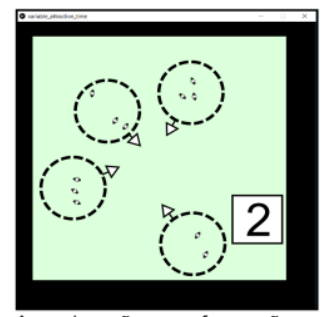
Aproximação para formação
de grupos

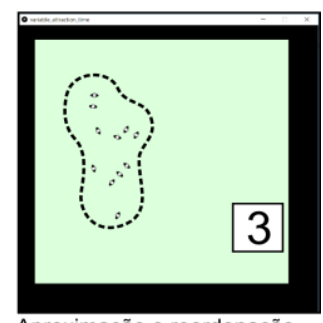

Aproximação e reordenação

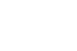

Fonte: Os autores.

Durante o processo de observação, foi verificado que os usuários, ao formarem a fila, também transformam o espaço em uma área de comunicação e troca de ideias, formando, assim, grupos.

Esse processo tem um impacto na sua configuração, sendo que os usuários não se alinham exatamente um atrás do outro, mas em pequenos grupos (Figura 7).

Como pode ser observado, na tela inicial da simulação automaticamente são gerados os agentes autônomos na configuração da fila, como descrito anteriormente (Figura 8). Além disso, também há a variação de luminosidade pela presença de nuvens ou não e da posição da sombra segundo o horário do dia. Os objetos que podem ser inseridos durante a simulação são as árvores, formando assim diferentes espaços de sombra. Os agentes são atraídos ou não segundo estas condições A Figura 9 ilustra uma simulação com a situação de formação de diferentes grupos de pessoas tanto no sol quanto na sombra e a alteração da dinâmica por meio da variação da nebulosidade e temperatura. Quando a temperatura está elevada, os agentes tendem a buscar a sombra gerada pela copa das árvores, caso o céu não esteja encoberto (incidência direta de luz solar). Esta situação pode ser observada na imagem 4. Por outro lado, verifica-se na imagem 5 , da mesma Figura, agentes na em espaços sem sombra, pois a simulação indica temperatura baixa, diminuindo a atratividade por sombra. Outros exemplos são mostrados na mesma Figura 9. 
Figura 9 - Implementação da simulação com a fila, formação de grupos, adição de árvores formando diferentes cenários. As imagens receberam símbolos adicionais para permitir uma leitura adequada

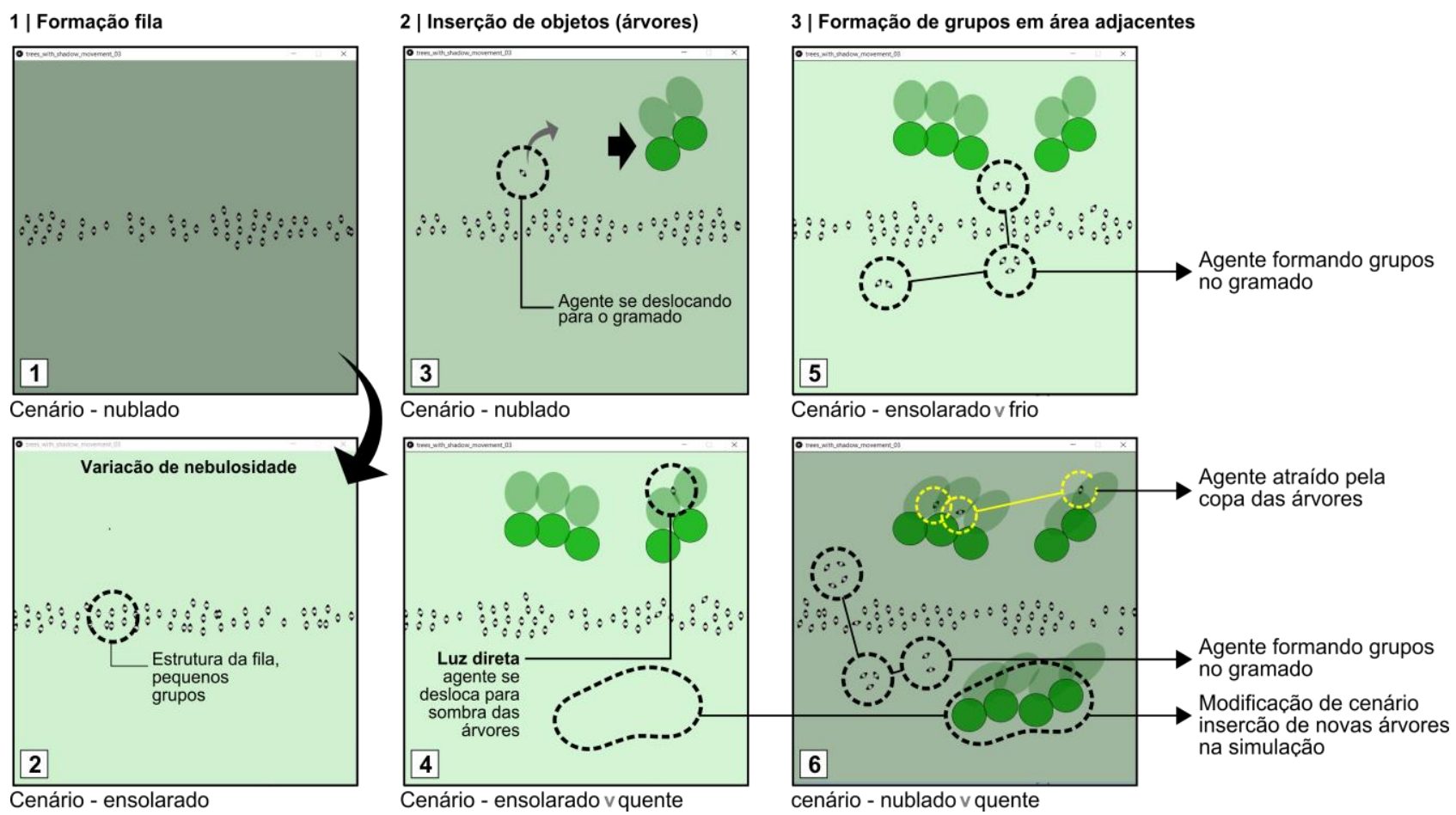

\section{Desdobramentos}

A partir da implementação dos agentes nesta pesquisa, pretende-se também aplicar a plataforma desenvolvida no ensino de arquitetura. A mesma simulação foi utilizada para implementar um protótipo de um sistema de iluminação. Nesse caso, os agentes foram empregados para simular a interação dos usuários com elementos luminosos interativos. O protótipo apresenta uma parte física, desenvolvida com o auxílio de uma placa de controle numérico que aciona uma série de LEDs segundo a proximidade dos agentes. A Figura 10 apresenta as diferentes etapas de implementação do sistema.

\section{Conclusões}

A partir do desenvolvimento deste trabalho de pesquisa foi possível criar uma sistemática de coleta de dados e representação de padrões por meio de regras esquemáticas. Em ambos os casos de estudo as regras são capazes de retratar de forma simples o processo de ocupação dos espaços. O conjunto de regras do espaço livre foi parcialmente implementado em uma simulação em um ambiente bidimensional. A implementação, como foi explicado, permite a inserção de representações de árvores e bancos. Contudo, a simulação se concentrou apenas na atração dos agentes entre si, formando grupos ou se aproximando ou afastando de áreas sombreada, dependendo dos parâmetros de temperatura e situações de céu coberto e encoberto. Apesar disso, foi possível representar algumas das dinâmicas de uso, ainda que apenas mais relacionadas com o fluxo de usuários.

Durante ambos os levantamentos também foi possível verificar a relação de usuários mais focados aos objetos presentes no espaço. Uma das relações que despertou mais interesse dos pesquisadores foi em relação às árvores no espaço livre. Nesse caso, notaram-se padrões de disposição do usuário em relação à sombra gerada pelas copas 
e o uso de objetos no espaço livre. Esse tipo de dinâmica mais focada será importante para futuramente enriquecer a simulação com agentes.

Figura 10 - Protótipo físico/digital desenvolvido por estudante para seu trabalho de conclusão de curso

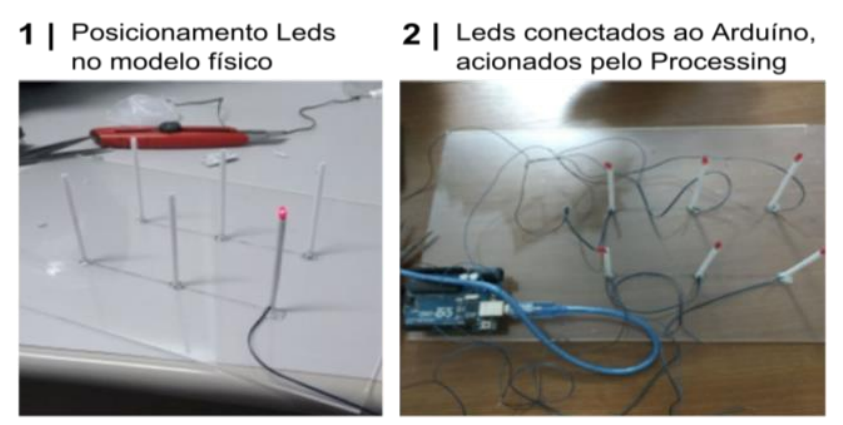

3 | Protótipo do sistema, modelo físico e implementação de agentes em Processing

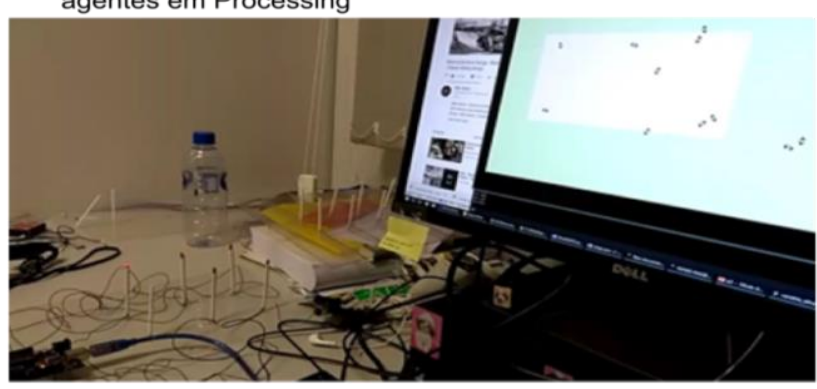

4| Sobreposição do modelo físico/digital

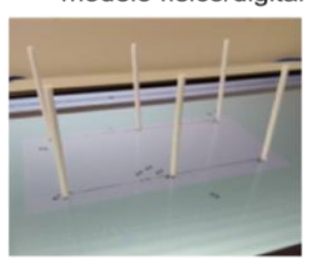

5| Montagem final do sistema

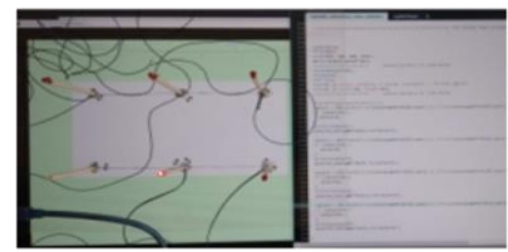

Fonte: Os autores.

Apesar de a simulação ter sido gerada em um ambiente bidimensional, a falta da terceira dimensão não torna esta inadequada. Atualmente existem diversas plataformas de jogos ou de renderização tridimensional que geram cenários mais reais. No entanto, não há por trás da programação desses sistemas uma preocupação em relação a como se comportam os agentes, principalmente em relação às situações de encontro, estar ou com os demais objetos presentes na simulação. O ambiente de programação Processing, portanto, mostrou-se adequado para a implementação da simulação, evitando que informações desnecessárias fossem adicionadas, desviando o objetivo inicial da pesquisa.

O processo de coleta de dados e sua sistematização em regras esquemáticas foram um passo importante para gerar posteriormente as simulações, pois estas foram suficientes para descrever de forma objetiva todas as informações coletadas durante a etapa de levantamento de dados. Sem esse processo de simplificação provavelmente haveria muito mais dificuldade para se transpor as dinâmicas na simulação.

Apesar disso, é importante destacar que a geração de uma simulação confiável depende do tempo de observação. Ou seja, quanto mais tempo os pesquisadores se debrucem no conhecimento de um espaço, mais padrões serão observados e, consequentemente, maior será a possibilidade de se criar uma simulação mais próxima da realidade. No entanto, é importante destacar que há uma dificuldade para se transpor as regras para 
o ambiente de programação, sendo necessário elencar quais são os padrões prioritários a serem implementados. Neste caso, como foi visto, voltou-se inicialmente às dinâmicas de fluxos, geração da relação entre agentes e áreas sombreadas etc.

Futuramente, além de enriquecer a simulação, pretende-se desenvolver métodos para a utilização desta plataforma como ferramenta de ensino de arquitetura paisagística.

\section{Agradecimentos}

À Capes pelo financiamento de uma bolsa de pesquisa de iniciação científica e ao PETARQ pela disponibilização de dois graduandos que colaboraram no desenvolvimento do trabalho.

\section{Referências}

BRAUN, A.; MUSSE, S. R.; OLIVEIRA, L. P. L. BODMANN, BARDO E. J. Modeling individual behaviors in crowd simulation. In: INTERNATIONAL CONFERENCE ON COMPUTER ANIMATION AND SOCIAL AGENTS, 16, 2003, New Brunswick. Proceedings [... ], New Brunswick: IEEE, 2003, p.143. DOI:https://doi.org/10.1109/CASA.2003.1199317

BUCKLAND, M. Al game programming by example. Tex.: Wordware, 2004.

GEHL, J. Life Between buildings: using public spaces. Washington: Island press, 2006.

GEHL, J.; SVARRE, B. How to study public space. Washington: Island Press, 2013.

GIBSON, J. The ecological approach to visual perception. Londres: Routledge. 1986.

GOFFMAN, E. Relations in Public: Microstudies of the Public Order. Nova York: Basic Books, 1971.

FRIEDENBERG, J.; SILVERMAN, G. Cognitive science: an introduction to the study of mind. California.: Sage Publications, 2006.

GRAHNA, P.; STIGSDOTTER, U. K. The relation between perceived sensory dimensions of urban green space and stress restoration. Landscape and Urban Planning, v. 94, Issues 3-4, p. 264-275, 2010.

DOI:https://doi.org/10.1016/j.landurbplan.2009.10.012

KAPLAN, R.; KAPLAN, S. The experience of nature: a psychological perspective. New York: Cambridge University Press, 1989.

KENDON, A. Conducting interaction: patterns of behavior in Focused Encounters. Nova York: Cambridge University Press, 1990.

LAMBERTS, R.; HENSENAND, J. L. M. Building performance simulation for design and operation. New York: Spon Press, 2011.

LAWSON, B. Language of Space. Oxford: Architectural, 2001.

MARSHALL, P; ROGERS, Y; PANTIDI, N. Using F-formations to analyse spatial patterns of interaction in physical environments. In: THE 2011 ACM CONFERENCE ON COMPUTER SUPPORTED COOPERATIVE WORK, 14., 2011, Hangzhou, China. Proceedings [...]. Nova York: CSCW 11 Conference Committee, 2011, p. 445-454. DOI:https://doi.org/10.1145/1958824.1958893

MITCHELL, W. J. The logic of architecture. Cambridge, Mass.: MIT Press,1990.

SIMEONE, D.; KALAY, Y. E. An Event-Based Model to simulate human behaviour in built environments. In: ANNUAL CONFERENCE OF EDUCATION AND RESEARCH IN COMPUTER AIDED ARCHITECTURAL DESIGN IN EUROPE, 30., 2012, Praga. Proceedings [... ]. Praga: ECAADE, 2012, v.1, p. 525-532. 
VAZ, Carlos Eduardo Verzola et al..

A interação de usuários em espaços livres: simulações com agentes autônomos

STINY, G. Shape: talking about seeing and doing. Cambridge, Massachusetts: MIT Press, 2006.

STINY, G.; GIPS, J. Shape grammars and the generative specification of paintings and sculpture. In C. V. Freiman, ed. Information processing 71, Amsterdam, 1972.

STINY, G. Pictorial and Formal Aspects of Shapes and Shape Grammars. Suiça: Birkhauser, 1975.

SHIFFMAN, D. The nature of code. California: Creative Commons, 2012.

VEITCH, R.; ARKKELIN, D. Environmental psychology: an interdisciplinary perspective. New Jersey: PrenticeHall, 1995.

WHYTE, W. H. The Social Life of Small Urban Spaces. Nova York: Project for Public Spaces,1971.

\section{Carlos Eduardo Verzola Vaz}

Arquiteto e Urbanista. Doutor. Docente no Departamento de Arquitetura e Urbanismo da Universidade Federal de Santa Catarina. Endereço postal: R. Eng. Agronômico Andrei Cristian Ferreira, s/n - Trindade, Florianópolis - SC, 88040-970.

\section{João Pedro Schneider}

Estudante de Arquitetura e Urbanismo. Estudante de graduação. R. Eng. Agronômico Andrei Cristian Ferreira, s/n Trindade, Florianópolis - SC, 88040-970.

\section{Marcus Vinícius da Silva}

Estudante de Arquitetura e Urbanismo. Estudante de graduação. R. Eng. Agronômico Andrei Cristian Ferreira, s/n Trindade, Florianópolis - SC, 88040-970.

\section{Renata Nunes Brochi Rodrigues}

Estudante de Arquitetura e Urbanismo. Estudante de graduação. R. Eng. Agronômico Andrei Cristian Ferreira, s/n Trindade, Florianópolis - SC, 88040-970.

\section{Arthur Stofella}

Arquiteto e Urbanista. Bacharel. R. Eng. Agronômico Andrei Cristian Ferreira, s/n - Trindade, Florianópolis - SC, 88040-970. 